\title{
Drying Method, Not Otherwise Specified
}

National Cancer Institute

\section{Source}

National Cancer Institute. Drying Method, Not Otherwise Specified. NCI Thesaurus. Code C112978.

A process that is not otherwise specified, which removes water or a volatile solvent by evaporation. 